\title{
Study of the Local Environment of Mn Ions Implanted in $\mathrm{GaSb}$
}

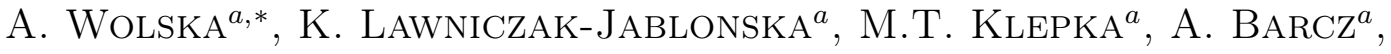 \\ A. HALlEN ${ }^{b}$ AND D. ARVANITIS ${ }^{c}$ \\ ${ }^{a}$ Institute of Physics PAS, al. Lotników 32/46, 02-668, Warsaw, Poland \\ ${ }^{b}$ Royal Inst. of Technology, Dept. of Microelectronics and Applied Physics \\ P.O. Box Electrum 226, 16440 Kista, Sweden \\ ${ }^{c}$ Physics Department, Uppsala University, Box 530, 75121 Uppsala, Sweden
}

\begin{abstract}
The first attempts to establish an implantation process leading to formation of ferromagnetic inclusions inside the GaSb matrix are presented. Gallium antimonide containing ferromagnetic MnSb precipitations is considered as a promising material for novel spintronic applications. It is possible to obtain such inclusions during the molecular beam epitaxy (MBE) growth. However, for commercial application it would be also important to find an optimal way of producing this kind of inclusions by Mn ions implantation. In order to achieve this goal, several parameters of implantation and post annealing procedures were tested. The ion energy was kept at $10 \mathrm{keV}$ or $150 \mathrm{keV}$ and four different ion doses were applied, as well as various annealing conditions. The analysis of X-ray absorption spectra allowed to estimate the local atomic order around Mn atoms. Depending on the implantation energy and annealing processes, the manganese oxides or manganese atoms located in a heavily defected GaSb matrix were observed. The performed analysis helped in indicating the main obstacles in formation of $\mathrm{MnSb}$ inclusions inside the GaSb matrix by $\mathrm{Mn}$ ion implantation.
\end{abstract}

PACS numbers: 78.70.Dm, 61.72.U-, 61.05.cj

\section{Introduction}

GaMnAs is considered as a promising material for novel spintronic applications. The uniform ternary alloys as well as related material containing inclusions of ferromagnetic MnAs have been intensively investigated. It was found, in particular, that nanosized MnAs ferromagnets buried in GaAs can be obtained not only by the MBE method, but also by Mn ion implantation into GaAs wafers [1-11]. Different types of magnetic inclusions $\left(\mathrm{Ga}_{x} \mathrm{Mn}_{y}, \mathrm{MnAs}\right.$ hexagonal and GaMnAs cubic) with a variety of magnetic properties were formed depending on the applied Mn ion energy, dose and post implantation annealing procedures. However, other types of inclusions, like MnSb, also show promising magnetic properties at room temperature. It is possible to obtain such inclusions during the MBE growth [12]. On the other hand, the implantation method is also well established, but cheaper and easier to carry out within the industrial production process. Therefore, it would be important to find an optimal way of producing the $\mathrm{MnSb}$ inclusions with desirable properties by $\mathrm{Mn}$ ions implantation, as it has been done in the MnAs case. To our knowledge there are no reports in the literature about

* corresponding author; e-mail: wolska@ifpan.edu.pl
Mn inclusions formed in GaSb by implantation. Therefore, we applied a low and high energy implantation with several Mn doses and performed several heat treatments to exam the influence of these parameters on the $\mathrm{Mn}$ containing inclusions. The X-ray absorption technique was applied to determine the local atomic order around implanted Mn atoms.

\section{Experiment}

Two sets of GaSb (100) crystals implanted with Mn ions are presented. The implantation processes were carried out at nominal room temperature with a beam current of a few $\mu \mathrm{A}$. The beam was raster scanned vertically and horizontally with frequencies of $64 \mathrm{~Hz}$ and $517 \mathrm{~Hz}$, respectively, covering a total area of about $20 \mathrm{~cm}^{2}$.

The first set of samples is called "low doses and energy" where the implantation energy was equal to $10 \mathrm{keV}$ and the doses of $\mathrm{Mn}$ ions were as follow: dose1 = $1 \times 10^{16} \mathrm{Mn} / \mathrm{cm}^{2}$, dose $2=2 \times 10^{16} \mathrm{Mn} / \mathrm{cm}^{2}$ and dose $3=$ $3 \times 10^{16} \mathrm{Mn} / \mathrm{cm}^{2}$. After the implantation all the samples were annealed in a vacuum furnace at $650^{\circ} \mathrm{C}$ for $10 \mathrm{~min}$.

Second set of the samples is called "higher dose and energy". In this case the implantation energy was equal to $150 \mathrm{keV}$ and the dose was the same $\left(1.7 \times 10^{17} \mathrm{Mn} / \mathrm{cm}^{2}\right)$ for the whole set. The time to reach the dose was seven hours and under these circumstances, the heating by the 
beam is not expected to raise the temperature more than about $10^{\circ} \mathrm{C}$. One of the samples, as-implanted, was left without any additional treatment. The next one, Ar350 was annealed in $\mathrm{Ar}$ atmosphere at $350^{\circ} \mathrm{C}$ for $5 \mathrm{~min}$. Two others were annealed in vacuum with $\mathrm{Sb}$ vapors at $400^{\circ} \mathrm{C}$ for $48 \mathrm{~h}(\mathrm{Sb} 400)$ and at $600^{\circ} \mathrm{C}$ for $2 \mathrm{~h}(S b 600)$.

$\mathrm{X}$-ray absorption near edge structure (XANES) and extended X-ray absorption fine structure (EXAFS) measurements at the $K$ edge of Mn were performed at DESY-Hasylab (Cemo and E4 stations) at liquid nitrogen temperature in a fluorescence mode using a 7-element silicon detector.

In order to facilitate XANES analysis, the ab-initio calculations using FEFF 8.4 code [13] were carried out. This kind of approach is very helpful in cases when the localization of a dopant is considered and the standard samples do not exist. The used procedure was as follow. A cluster of $10 \AA$ in radius was first created using the known crystallographic data for GaSb structure [14] and then, after the modifications, the XANES spectra were calculated using the XANES, self-consistent field (SCF) and full multiple scattering (FMS) cards. The HedinLundqvist potential was chosen.

EXAFS analysis was conducted using IFEFFIT data analysis package with Athena and Artemis programs [15]. The EXAFS measurements were carried out up to $\approx 655 \mathrm{eV}$ above the edge. The region chosen to perform a Fourier transformation was $3.2-10.4 \AA^{-1}$. It gives around 15 independent points, which was always considerably more than the number of variables used in the fitting.

To check the influence of different treatment on the distribution of atoms in crystals, the secindary ion mass spectroscopy (SIMS) measurements using a CAMECA IMS6F micro-analyzer were also carried out.

\section{Results and discussion}

\subsection{Low doses and energy}

GaSb (100) crystals implanted with Mn ions to a dose1 $\left(1 \times 10^{16} \mathrm{Mn} / \mathrm{cm}^{2}\right)$, dose $2\left(2 \times 10^{16} \mathrm{Mn} / \mathrm{cm}^{2}\right)$ and dose 3 $\left(3 \times 10^{16} \mathrm{Mn} / \mathrm{cm}^{2}\right)$ are first considered. Increase of the absorption jump in XANES spectra for higher doses is consistent with the higher Mn concentration (Fig. 1). The shapes of the spectra after normalization are very similar, which suggests that $\mathrm{Mn}$ atoms are located in similar positions independently of the dose. Comparison with the spectrum of the standard MnSb sample excluded possibility of MnSb formation in implanted samples (Fig. 2).

In order to check possible Mn atoms locations, theoretical XANES spectra were calculated using FEFF8.4 code. The GaSb crystallographic data were used to create a cluster consisting of all the atoms within a $10 \AA$ radius from the central atom. Then, the Mn atoms were introduced into the matrix in the substitutional ( $\mathrm{Ga}$ or $\mathrm{Sb}$ ) or interstitial positions by adding the Mn atoms into the cluster. In Fig. 2 the results of calculations for Mn substituting $\mathrm{Ga}\left(\mathrm{Mn}_{\mathrm{Ga}}\right.$ in $\left.\mathrm{GaSb}\right)$ or $\mathrm{Sb}\left(\mathrm{Mn}_{\mathrm{Sb}}\right.$ in $\left.\mathrm{GaSb}\right)$ are

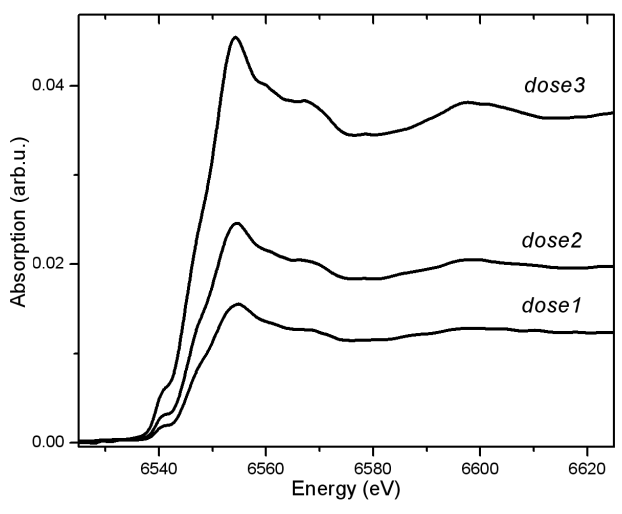

Fig. 1. XANES measurements at the manganese $K$ edge of the samples implanted with low energy ions and with different doses.

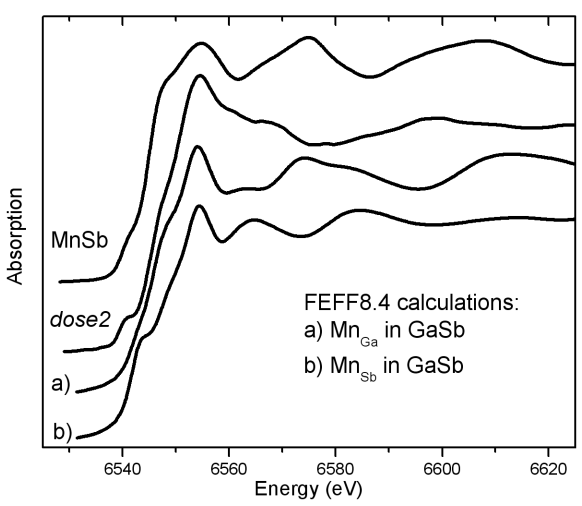

Fig. 2. Experimental XANES spectrum of dose2 versus FEFF8.4 calculations for substitutional Mn positions in the GaSb matrix: (a) $\mathrm{Mn}_{\mathrm{Ga}}$, (b) $\mathrm{Mn}_{\mathrm{Sb}}$. XANES of the MnSb standard is shown for comparison.

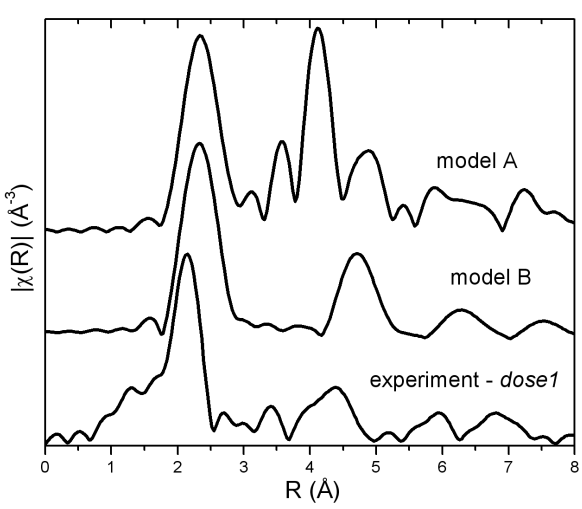

Fig. 3. The Fourier transform of the EXAFS spectrum of dose 1 sample and theoretical models based on $\mathrm{GaSb}$ crystal with Mn substituting Sb. Model A: full GaSb matrix. Model B: the Ga sublattice only. Parameters used to calculate the models: $S_{0}^{2}=0.7, \sigma^{2}=1$ st shell 0.003 , 2nd 0.005 , 3rd 0.007, 4th 0.009 . 


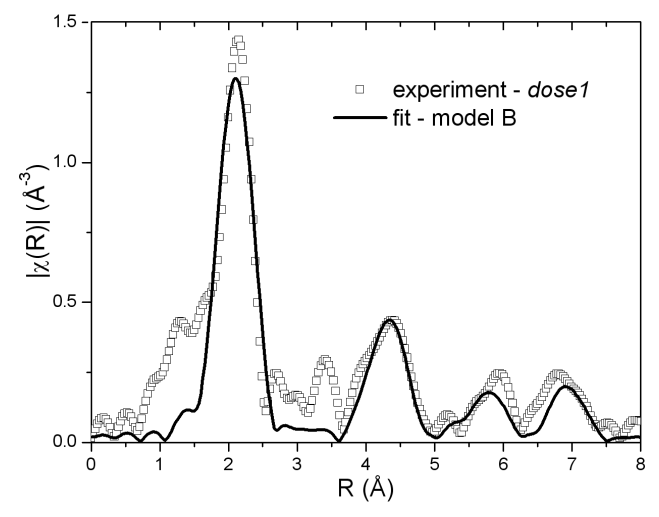

Fig. 4. Fitting of the EXAFS spectrum of dose1 sample using the single scattering Ga paths for four consecutive Ga spheres in the GaSb crystal (model B).

shown together with the experimental spectrum. The shapes do not agree with the experiment, therefore, we can draw the conclusion that Mn atoms do not locate in subtitutional positions in the GaSb crystal. Several models for the interstitial positions are not presented here because none of them gave better results. This means that none of the models consisting of $\mathrm{Mn}$ atoms located in the $\mathrm{GaSb}$ matrix is correct in this case.

EXAFS analysis usually provides more detailed information about the distribution of atoms around the selected element. Here, again we start from the GaSb model. In Fig. 3 the magnitude of Fourier transformed EXAFS oscillations of one of the samples and two theoretical models are presented. Model A is a sum of 8 single scattering paths corresponding to the consecutive $4 \mathrm{Ga}$ and $4 \mathrm{Sb}$ coordination spheres in the GaSb crystal, where the central $\mathrm{Mn}$ atom is located in the $\mathrm{Sb}$ position. The number of atoms in each coordination sphere was kept set according to the GaSb crystallographic data. Model B also presents a sum of scattering paths in the GaSb matrix, but it takes into account only scatterings on the Ga atoms, i.e., 4 single scattering Ga paths corresponding to four consecutive shells consisting of Ga atoms. Simple comparison demonstrates that model A differs considerably from the experiment which suggests that there is a deficit of $\mathrm{Sb}$ atoms in the Mn neighbourhood. In case of model $\mathrm{B}$, without the $\mathrm{Sb}$ atoms, the result seems to resemble the experimental spectrum better, but distances to the subsequent shells are too large. However, with a lack of antimony atoms, it can be expected that remaining Ga atoms rearrange their positions and they move closer to the central atom. In order to check the validity of these considerations, fitting of the experimental spectrum was performed.

The $\Delta R$ parameter responsible for monitoring the changes in distances $(R)$ was defined as $R * \alpha$, where $\alpha$ is a measure of the lattice contraction. For the sample implanted with the lowest dose the structure is most regular. Four consecutive Ga shells can be distinguished and four single scattering Ga paths (with the degeneracy determined by the crystallographic data) were used in the fitting. The result is shown in Fig. 4. The model works reasonably well even up to $8 \AA$. The fit is not perfect because only single scattering Ga paths were considered, but it clearly illustrates the behaviour of the Mn neighbourhood. During the implantation the Sb sublattice around Mn atoms is destroyed and it is not reconstructed during annealing procedure, in contrary to the Ga sublattice which only contracts significantly. The comparison between theoretical distances to the subsequent shells and those found from the fitting are shown in Table I.

TABLE I

Results of fitting the EXAFS spectra of dose1 sample with a single scattering Ga paths for four subsequent Ga coordination spheres.

\begin{tabular}{c|c|c|c|c}
\hline \hline & I shell $(4 \mathrm{Ga})$ & II shell $(12 \mathrm{Ga})$ & III shell $(12 \mathrm{Ga})$ & IV shell $(16 \mathrm{Ga})$ \\
\hline$R_{\mathrm{Ga}}[\AA]($ model $)$ & 2.65 & 5.07 & 6.66 & 7.95 \\
\hline$R_{\mathrm{Ga}}[\AA](\mathrm{fit})$ & 2.43 & 4.66 & 6.12 & 7.30 \\
\hline$\Delta R$ & 0.18 & 0.41 & 0.54 & 0.65 \\
\hline$\sigma^{2}\left[\AA^{2}\right]$ & $0.007 \pm 0.001$ & $0.011 \pm 0.002$ & $0.012 \pm 0.003$ & $0.010 \pm 0.002$
\end{tabular}

In Fig. 5 the results of fitting for low energy samples with all doses are shown. Only two Ga shells were considered due to a fact that for higher doses the structure above $5 \AA$ from Mn atoms was not well preserved. It indicates that an increase of dose introduces higher disorder in the sample. Both shells consist of gallium atoms, however, some residual antimony atoms are also found between the shells. In such a case, because of correlations between the parameters, it is not possible to determine accurately how many of the Sb atoms are located in there. Therefore, the number of additional Sb atoms was kept at the same level in all fits and $\sigma_{\mathrm{Sb}}^{2}$ parameter 


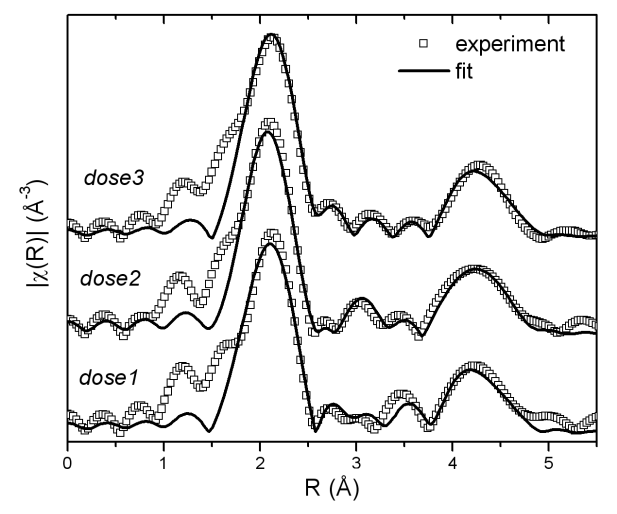

Fig. 5. Fitting results of EXAFS spectra for dose1, dose2 and dose3 samples, up to the second coordination shell. Residual Sb atoms were added.

(XAFS Debye-Waller factor for the Sb shells) was used to monitor changes in the structural disorder. Its high values for the investigated samples indicate that, in average, there are less antimony atoms for each central Mn atom than it was assumed. Values of the fitting parameters are presented in Table II.

TABLE II

Results of fitting the EXAFS spectra of "low doses and energy" samples. $S_{0}^{2}$ represents an amplitude attenuation factor, $\sigma^{2}$ - XAFS Debye-Waller factor, and $R$-factor is a measure of the quality of the fit.

\begin{tabular}{c|c|c|c}
\hline \hline & dose1 & dose2 & dose3 \\
\hline$S_{0}^{2}$ & $0.46 \pm 0.12$ & $0.50 \pm 0.13$ & $0.54 \pm 0.11$ \\
\hline$\sigma_{\mathrm{Ga} 1}^{2}\left[\AA^{2}\right]$ & $0.003 \pm 0.002$ & $0.003 \pm 0.002$ & $0.003 \pm 0.002$ \\
\hline$\sigma_{\mathrm{Ga} 2}^{2}\left[\AA^{2}\right]$ & $0.007 \pm 0.002$ & $0.007 \pm 0.002$ & $0.008 \pm 0.002$ \\
\hline$\alpha$ & $-0.088 \pm 0.004$ & $-0.087 \pm 0.004$ & $-0.084 \pm 0.003$ \\
\hline$R_{\mathrm{Ga} 1}[\AA]$ from $\alpha$ & 2.42 & 2.42 & 2.43 \\
\hline$R_{\mathrm{Ga} 2}[\AA]$ from $\alpha$ & 4.63 & 4.63 & 4.65 \\
\hline$R_{\mathrm{Sb} 1}[\AA](1.5$ at. $)$ & $2.76 \pm 0.06$ & $2.78 \pm 0.05$ & $2.87 \pm 0.11$ \\
\hline$R_{\mathrm{Sb} 2}[\AA](2$ at. $)$ & $3.80 \pm 0.06$ & $3.06 \pm 0.04$ & $3.76 \pm 0.12$ \\
\hline$\sigma_{\mathrm{Sb}}^{2}\left[\AA^{2}\right]$ & $0.008 \pm 0.006$ & $0.007 \pm 0.004$ & $0.025 \pm 0.016$ \\
\hline$R$-factor & 0.069 & 0.048 & 0.040
\end{tabular}

In summary, EXAFS analysis showed that even low energy implantation and a quick annealing lead to the significant deficit of $\mathrm{Sb}$ atoms around implanted $\mathrm{Mn}$ atoms. Moreover, Mn atoms are located in the antimony positions in the GaSb matrix where the $\mathrm{Sb}$ sublattice is almost completely destroyed, and the remaining Ga sublattice contracts significantly.

\subsection{GaMn models}

In case of the Mn implantation in the GaAs matrix, the formation of $\mathrm{Ga}-\mathrm{Mn}$ inclusions was reported in $[3,9,10]$. The inclusions were identified as $\mathrm{Ga}_{5} \mathrm{Mn}_{8}$ [3] or $\mathrm{Ga}_{3} \mathrm{Mn}_{2}$ [9] and showed ferromagnetic properties. However, the detailed analysis of their structure was not performed. To our knowledge, there is no report about the results of $\mathrm{Mn}$ implantation into GaSb crystals.
Analysis of the spectra of the samples implanted with low energy and low doses revealed that compound formed in the vicinity of $\mathrm{Mn}$ atoms up to few $\AA$ is in fact Ga:Mn, with the crystallographic order as in the space group $F-43 m$ characteristic for GaSb matrix. According to the crystallographic databases [16-20] none of the bulk GaMn or Ga compounds crystallizes in that structure. Performing the fitting, we considered the $\mathrm{Ga}_{y} \mathrm{Mn}_{x}$, as well as Ga structures. The results were negative for all of them. As the examples we decided to present the models of four $\mathrm{Ga}_{y} \mathrm{Mn}_{x}$ crystals with different space groups:

a) $F m-3 \mathrm{~m}, \mathrm{Ga}_{0.44} \mathrm{Mn}_{0.56}[17]$,

b) $P 6_{3} / m m c, \mathrm{GaMn}_{2}[18]$,

c) $R-3 m$, GaMn [19],

d) $I 4 / m m m, \mathrm{Ga}_{1.15} \mathrm{Mn}_{2.85}[20]$.

Attempts of the fitting using these models failed. The parameters of the fits were persistently unphysical. Therefore, only comparisons of theoretical curves with the experimental dose1 spectrum are presented in Figs. 6a-d. In each of the cases the clusters of atoms were created based on the crystallographic data and then, used in calculations of scattering paths. The parameters, like distances and number of atoms, were kept without modifications. Only the amplitude reduction factor $\left(S_{0}^{2}\right)$ and XAFS Debye-Waller factor $\left(\sigma^{2}\right)$ were adjusted manually in order to facilitate comparisons.
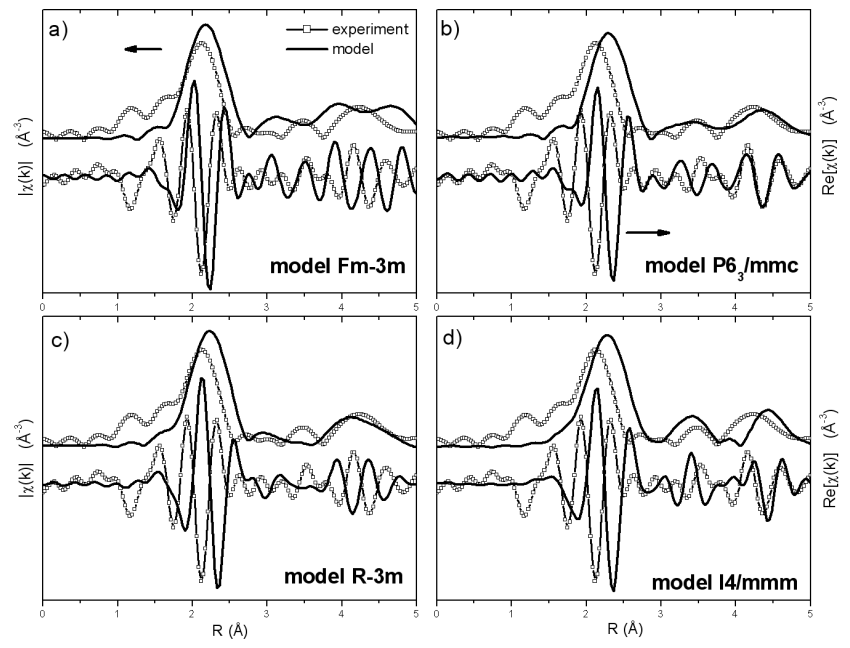

Fig. 6. The magnitudes (upper curves) and the real part of Fourier transforms (lower curves) obtained for considered models of crystal structures compared with experimental spectrum for dose 1 sample. The curves were arbitrary shifted along the $y$-axis in order to make the plots more readable.

The Fm-3m model $\left(\mathrm{Ga}_{0.44} \mathrm{Mn}_{0.56}\right)$ is presented in Fig. 6a. Mn and Ga atoms share the same crystallographic position in this structure. For example, the first shell consists of 12 atoms (5.28 Ga and 6.72 Mn atoms in 
average). Since calculations of the scattering paths can not be performed for a site with partial occupancy, two clusters were constructed: one with only $\mathrm{Mn}$, and another with only Ga atoms. Then, scattering paths were calculated and summed together in proper proportions. The values of parameters used in modeling are: $S_{0}^{2}=0.3$, $\sigma^{2}=1$ st shell 0.005 and 0.008 in the subsequent shells.

The $P 6_{3} / m m c$ model $\left(\mathrm{GaMn}_{2}\right)$ is shown in Fig. 6b. As in the previous case, $\mathrm{Mn}$ and Ga atoms share the same crystallographic position. The first shell consists of 12 atoms (3.96 $\mathrm{Ga}$ and $8.04 \mathrm{Mn}$ atoms in average). Again, two clusters were constructed and the Mn and Ga paths were summed together in proper proportions. The values of parameters used in modeling are: $S_{0}^{2}=0.3, \sigma^{2}=1$ st shell 0.005 and 0.008 in the subsequent shells.

The $R-3 m$ model (GaMn) is presented in Fig. 6c. Mn atoms occupy three non-equivalent crystallographic positions. All of them were taken into consideration in correct proportions. The first and second shells consist of a mixture of $\mathrm{Ga}$ and $\mathrm{Mn}$ atoms. The values of parameters used in modeling are: $S_{0}^{2}=0.6, \sigma^{2}=1$ st shell $0.005,2$ nd shell 0.006 .

The $I 4 / \mathrm{mmm}$ model $\left(\mathrm{Ga}_{1.15} \mathrm{Mn}_{2.85}\right)$ is shown in Fig. 6d. Mn atoms occupy two non-equivalent crystallographic positions. Again, both of them were used in the summation. All shells consist of a mixture of Ga and $\mathrm{Mn}$ atoms. The values of parameters used in modeling are: $S_{0}^{2}=0.6, \sigma^{2}=1$ st shell 0.008 and 0.006 in the subsequent shells.

As it can be seen in Figs. 6a-d, magnitudes of the Fourier transforms of the models can be reasonably close to the experimental spectrum of "low doses and energy" samples. However, in all the cases, the phase of oscillations in the real part of the transform does not agree between the models and the experiment, which explains the problems encountered during the fitting. The local order around $\mathrm{Mn}$ dopants differs from that in $\mathrm{Ga}_{y} \mathrm{Mn}_{x}$ compounds reported in the literature.

\subsection{Higher dose and energy}

The XANES spectra of GaSb (100) crystals implanted with $\mathrm{Mn}$ ions with energy equal to $150 \mathrm{keV}$ and the dose of $1.7 \times 10^{17} \mathrm{Mn} / \mathrm{cm}^{2}$, compared with $\mathrm{MnSb}$ powder standard spectrum, excluded possibility of $\mathrm{MnSb}$ formation in this case as well. To obtain the information about the Mn location in the samples, EXAFS analysis was performed.

In the Fourier transformed EXAFS oscillations of as-implanted sample only the first shell is visible (Fig. 7a). This suggests that the Mn neighbourhood is rather amorphous. EXAFS analysis shows that this shell can consist of Ga and Mn atoms (Table III). The $S_{0}^{2}$ parameter in this case was set to the value found from the fitting of the annealed sample. Two atoms of $\mathrm{Mn}$ and 6 atoms of $\mathrm{Ga}$ were put in here arbitrary. However, the high value of $\sigma^{2}$ for Mn atoms indicates that the number of Mn neighbours is smaller. As it was expected, the implantation

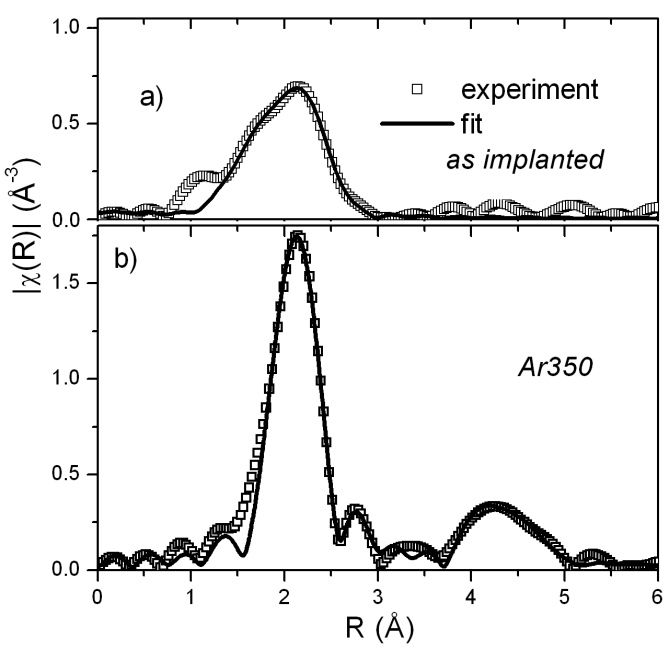

Fig. 7. Fitting result of EXAFS spectra for a high energy as-implanted sample and for the sample annealed in Ar atmosphere (Ar350).

with high energy ions leads to a lack of long range order around implanted ions.

TABLE III

Results of fitting the EXAFS spectra for high energy as-implanted and the annealed in Ar atmosphere (Ar350) sample. The third cumulant (3rd cum) describing an asymmetric non-Gaussian pair distribution function of atoms had to be introduced in $\mathrm{Ga}$ and $\mathrm{Mn}$ subshells in case of the as-implanted sample.

\begin{tabular}{c|c|c}
\hline \hline & as-implanted & Ar350 \\
\hline$S_{0}^{2}$ & 0.84 & $0.84 \pm 0.15$ \\
\hline$R_{\mathrm{Ga} 1}[\AA]$ & $2.57 \pm 0.11(6$ at. $)$ & $2.46 \pm 0.01(4$ at. $)$ \\
\hline$\sigma_{\mathrm{Ga} 1}^{2}\left[\AA^{2}\right]$ & $0.004 \pm 0.003$ & $0.003 \pm 0.002$ \\
\hline $3 \mathrm{rd}$ cum & $0.003 \pm 0.004$ & - \\
\hline$R_{\mathrm{Ga} 2}[\AA]$ & - & $4.61 \pm 0.03(8.5$ at. $)$ \\
\hline$R_{\mathrm{Ga} 2}[\AA]$ & - & $4.99 \pm 0.08(3.5$ at. $)$ \\
\hline$\sigma_{\mathrm{Ga} 2}^{2}\left[\AA^{2}\right]$ & - & $0.011 \pm 0.003$ \\
\hline$N$ & $\mathrm{Mn}(2$ at. $)$ & $\mathrm{Sb}(1 \mathrm{at})$. \\
\hline$R[\AA]$ & $2.29 \pm 0.16(\mathrm{Mn})$ & $2.80 \pm 0.04(\mathrm{Sb})$ \\
\hline$\sigma^{2}\left[\AA^{2}\right]$ & $0.020 \pm 0.007(\mathrm{Mn})$ & $0.005 \pm 0.003(\mathrm{Sb})$ \\
\hline $3 \mathrm{rd}$ cum & $0.001 \pm 0.001(\mathrm{Mn})$ & - \\
\hline$R$-factor & 0.007 & 0.032
\end{tabular}

After annealing in Ar atmosphere (Ar350 sample) the first and the second shell were reconstructed, but not to the GaSb structure (Fig. 7b, Table III). Mn atoms are no longer visible in the first shell. Ga atoms dominate in the neighbourhood forming contracted Ga lattice, as it was shown for the samples with low Mn doses. However, in this case the second shell consists of Ga atoms at slightly different distances. We can relate it to the fact that the crystalline structure was not fully reconstructed. Addi- 
tionally, the presence of residual $\mathrm{Sb}$ atoms at $2.8 \AA$ was detected.

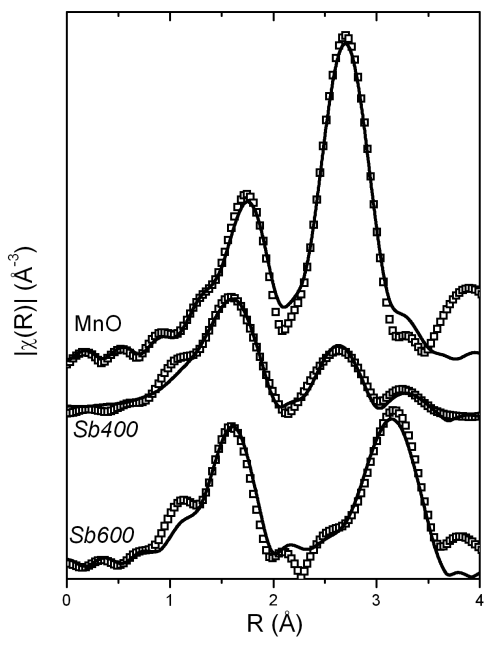

Fig. 8. Fitting result of EXAFS spectra for samples annealed in $\mathrm{Sb}$ vapours ( $S 6400$ and $\mathrm{Sb600)}$ ).

TABLE IV

Results of fitting the EXAFS spectra for samples annealed in Sb vapours $(S b 400, S b 600)$ and for the standard $\mathrm{MnO}$ powder. The values without estimated errors were kept set during the fitting.

\begin{tabular}{c|c|c|c}
\hline \hline & $\mathrm{MnO}$ & Sb400 & Sb600 \\
\hline$S_{0}^{2}$ & $0.69 \pm 0.07$ & 0.7 & 0.7 \\
\hline$R_{\mathrm{O}}[\AA]$ & $2.22 \pm 0.01$ & $2.09 \pm 0.01$ & $2.06 \pm 0.02$ \\
\hline$N_{\mathrm{O}}$ & 6 & $4.4 \pm 0.6$ & $3.5 \pm 0.8$ \\
\hline$\sigma_{\mathrm{O}}^{2}\left[\AA^{2}\right]$ & $0.008 \pm 0.002$ & $0.008 \pm 0.002$ & $0.003 \pm 0.003$ \\
\hline$R_{\mathrm{Mn}}[\AA]$ & $3.14 \pm 0.01$ & $3.12 \pm 0.02$ & $3.55 \pm 0.02$ \\
\hline$N_{\mathrm{Mn}}$ & 12 & $3.9 \pm 1.3$ & $11.5 \pm 3.3$ \\
\hline$\sigma_{\mathrm{Mn}}^{2}\left[\AA^{2}\right]$ & $0.007 \pm 0.001$ & $0.010 \pm 0.003$ & $0.009 \pm 0.003$ \\
\hline$R_{\mathrm{O} 2}[\AA]$ & $3.85 \pm 0.01$ & $3.88 \pm 0.04$ & \\
\hline$N_{\mathrm{O} 2}$ & 8 & $4.1 \pm 3.2$ & \\
\hline$\sigma_{\mathrm{O} 2}^{2}\left[\AA^{2}\right]$ & $0.008 \pm 0.002$ & $0.006 \pm 0.010$ & \\
\hline$R$-factor & 0.012 & 0.017 & 0.035
\end{tabular}

In samples annealed in Sb vapours completely different atomic order around $\mathrm{Mn}$ atoms was found. In this case, a high amount of oxygen was introduced during the heat treatment resulting in the formation of manganese oxides. Figure 8 and Table IV present the results of fitting of the MnO standard and of the investigated samples. The $\mathrm{S}_{0}^{2}$ parameter for $S b 400$ and $S b 600$ samples was set to the value found from the $\mathrm{MnO}$ fitting, what allowed to determine the number of the neighbours. In both samples the Mn atoms do not form a stoichiometric $\mathrm{MnO}$ with the long range order, but oxygen atoms are found in the first shell and different numbers of $\mathrm{Mn}$ atoms in the second one.

\subsection{SIMS results}

Distributions of the elements in "higher dose and energy" samples, measured by SIMS, are presented in Figs. 9a-d. SIMS data confirm that there is a depletion of the $\mathrm{Sb}$ atoms (in respect to $\mathrm{Ga}$ atoms) in the vicinity of the surface for as-implanted and Ar350 samples. In case of $S b 400$ and $S b 600$ samples the ratio of Ga to $\mathrm{Sb}$ atoms stays almost constant. It seems that annealing in $\mathrm{Sb}$ vapours keeps or reintroduces the $\mathrm{Sb}$ atoms in implanted samples. However, a high amount of oxygen was introduced during this procedure, what resulted in formation of $\mathrm{Mn}$ oxides. For as-implanted and Ar350 samples the level of oxygen atoms is much lower and the Mn oxides are below the level of detection by the EXAFS technique.

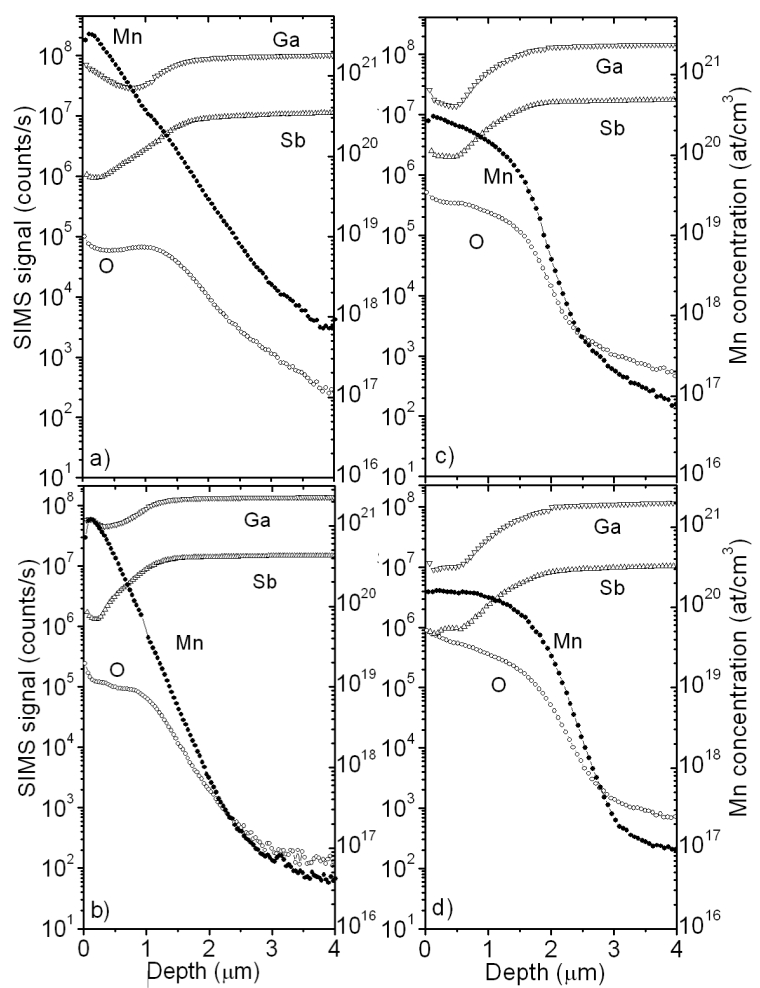

Fig. 9. SIMS depth profiles of the element distribution in the investigated high energy and high dose samples: (a) as-implanted, (b) Ar350, (c) Sb400, and (d) Sb600.

\section{Conclusions}

XANES measurements of the $K$ edge of Mn proved that the local structure around $\mathrm{Mn}$ atoms in considered samples does not resemble the MnSb structure. The EXAFS analysis allowed to determine the neighbourhood of Mn dopants introduced by the low and high energy implantation. Nevertheless, it should be stressed that this order exists only around Mn atoms and it is not valid for the whole volume of the sample. 
In summary, formation of the MnSb inclusions in $\mathrm{GaSb}$ substrates during $\mathrm{Mn}$ ion implantation encounters several obstacles. The implantation leads to removing the $\mathrm{Sb}$ atoms from the neighbourhood of $\mathrm{Mn}$ atoms even when the implantation temperature was kept close to room temperature. The annealing in Sb vapours reintroduced $\mathrm{Sb}$ atoms but together with the oxygen, which is more reactive and was bounded to Mn atoms. Therefore, the most difficult and important task is to prevent the removing of the Sb atoms during implantation.

$\mathrm{MnSb}$ precipitates were not formed in tested implantation processes. In most cases, inclusions containing Ga:Mn material were created. In these inclusions Mn atoms are located in the contracted Ga sublattice preserving a crystalline order characteristic for the GaSb crystal with the $F-43 m$ space group. This kind of crystal structure has not been observed before for $\mathrm{Ga}_{x} \mathrm{Mn}_{y}$ compounds.

\section{Acknowledgments}

Authors would like to thank Dr. Zbigniew Golacki for help in the thermal treatment of some of the samples. This work was partially supported by national grant of Ministry of Science and High Education N202-052-32 1189. The research leading to these results has received funding from the European Community's Seventh Framework Programme (FP7/2007-2013) under grant agreement no. 226716 .

\section{References}

[1] A. Chanda, H.P. Lenka, C. Jacob, Appl. Phys. A 94 89 (2009).

[2] O.D.D. Couto, Jr., M.J.S.P. Brasil, F. Iikawa, C. Giles, C. Adriano, J.R.R. Bortoleto, M.A.A. Pudenzi, H.R. Gutierrez, I. Danilov, Appl. Phys. Lett. 86, 071906 (2005)

[3] S.L. Song, N.F. Chen, J.P. Zhou, Z.G. Yin, Y.L. Li, S.Y. Yang, Z.K. Liu, J. Cryst. Growth 264, 31 (2004).
[4] M.A. Scarpulla, O.D. Dubon, K.M. Yu, O. Monteiro, M.R. Pillai, M.J. Aziz, M.C. Ridgway, Appl. Phys. Lett. 82, 1251 (2003).

[5] C. Chen, M. Cai, X. Wang, S. Xu, M. Zhang, X. Ding, Y. Sun, J. Appl. Phys. 87, 5636 (2000).

[6] J. Yang, N.F. Chen, Z. Liu, S. Yang, C. Chai, M. Liao, H. He, J. Cryst. Growth 234, 359 (2002).

[7] K. Ando, A. Chiba, H. Tanoue, Appl. Phys. Lett. 73, 387 (1998).

[8] P.J. Wellmann, J.M. Garcia, J.L. Feng, P.M. Petroff, Appl. Phys. Lett. 71, 2532 (1997).

[9] J. Shi, J.M. Kikkawa, D.D. Awschalom, G. Medeiro-Riberio, P.M. Petroff, K. Babcock, J. Appl. Phys. 79, 5296 (1996).

[10] J. Shi, J.M. Kikkawa, R. Proksch, T. Schaffer, D.D. Awschalom, G. Medelros-Ribeiro, P.M. Petroff, Nature (London) 377, 707 (1995).

[11] K. Ando, A. Chiba, H. Tanoue, F. Kirino, M. Tanaka, IEEE Trans. Magn. 35, 3463 (1999).

[12] E. Abe, F. Matsukura, H. Yasuda, Y. Ohno, H. Ohno, Physica E 7, 981 (2000)

[13] A.L. Ankudinov, B. Ravel, J.J. Rehr, S.D. Conradson, Phys. Rev. B 58, 7565 (1998).

[14] R.W.G. Wyckoff, Crystal Structures 1, 85-237 (1963) Second edition. Interscience Publishers, New York.

[15] B. Ravel, M. Newville, J. Synchrotron Rad. 12, 537 (2005).

[16] American Mineralogist Crystal Structure Database: http://rruff.geo.arizona.edu/AMS .

[17] K.H.J. Buschow, P.G. van Engen, R. Jongebreur, J. Magn. Magn. Mat. 38, 1 (1983); Inorganic Crystal Structure Database \#103797.

[18] H.G. Meissner, K. Schubert, Zeitschrift fuer Metallkunde 56, 523 (1965); Inorganic Crystal Structure Database \#103798.

[19] O. Gourdon, G.J. Miller, J. Sol. Stat. Chem. 173 , 137 (2003); Inorganic Crystal Structure Database \#96491.

[20] E. Kren, G. Kadar, Sol. Stat. Commun. 8, 1653 (1970); Inorganic Crystal Structure Database \#103799. 